\title{
Analysis on Cultural Characteristics and Fitness Value of Tujia Waving Dance
}

\author{
Zeng Zheng \\ School of Music, China West Normal University, \\ Nanchong, 637000, China
}

\begin{abstract}
As a kind of national culture, the national dance is an aggregation of integrated environmental factors; it gradually derives from people's daily life, and then it is endowed with national cultural spirit and becomes the natural ecology and social ecology unique to one nationality. Tujia waving dance is the product of Tujia cultural and ecologic environment, and it is closely related to the regional cultural elements. From perspective of its social function, except for social communication, it also exerts the fitness function; if we carry out further investigation on Tujia waving dance from perspective of folk custom, we can find that the it has good ornamental value and fitness value in terms of its body movement way. This paper carries out a research on cultural characteristics and fitness value of Tujia waving dance.
\end{abstract}

Key words: Tujia waving dance; intangible cultural heritage; cultural characteristics; social function; fitness value

China has vast territory and abundant resources as well as rich cultural heritage. As a multinational country, each nationality creates the culture which its unique style, which adds strong and bright color on China's national culture. Tujia people inhabits southwest China. As an important part of Chinas minorities, various kinds of folk custom activities of Tujia nationality have become the important content of Chinese folk custom research, and Tujia dance is also an important folk custom research content. Because Tujia people are always under closed and semi-closed state, their living state is not deeply excavated. However, we can get a knowledge of historic culture of this nationality via research on Tujia waving dance, which is of important significance for research on Tujia folk culture.

\section{Origin of Tujia waving dance}


Tujia people have their own folk-custom activities, such as sacrifice, social contact, and sports events, all of which are used as Tujia people’s way of communication in daily life. Currently, some of Tujia people’s folk-custom activities have been listed into Chinese intangible cultural heritage. As for research on Tujia waving dance, the key is to make investigation on dance form, and the origin of waving dance remains to be further explored. The main reason is that there are no Tujia characters which come down, thus there lacks of character record. Therefore, we shall carry out investigation on cultural characteristics and functional value of waving dance, and then extend to social and historic culture of Tujia nationality so as to deeply dig out Tujia people's living state and then research the social historicality of waving dance.

In Tujia nationality, the "waving” is called "She Ba”. Tujia people call "waving dance” as "She Ba Ri” in which the "Ri” means “do”. In terms of grammatical structure, Tujia language follows the rule of verb-object inversion; therefore, "She Ba Ri” is translated as "do waving” in Chinese; in order to express the language clearly, "She Ba Ri" is translated as "waving dance”. As the traditional song and dance of Tujia nationality, the "waving dance” is also called "She Ba Ba”, and "Mao Tuo Ge Ci”. While the song and dance activity is carried out, people pay great attention to ritual and etiquette; according to the cultural tradition of Tujia nationality, the waving dance is mainly held in the $3^{\text {rd }}$ day of lunar January. Currently, as a traditional entertainment activity, the Tujia "waving dance" is often made in odd-numbered day; the activity is generally continued for 3 days and 3 nights, and it can be ended also in odd-numbered day.

Tujia people in southwest China mainly live in 8 counties (Longshan, Guzhang, Dayong, Yongshun, Baojing, Sangzhi, Huayuan, and Jishou), and the waving dance activities are commonly made in those regions. Especially in the Spring Festival of Tujia nationality, the waving dance will be made as a group activity. Tujia people and Han people spend respective Spring Festival in different days; Tujia people’s Spring Festival will be generally 1 or 2 days in advance or even 1 week in advance, and the "waving dance”, as a traditional cultural activity which is passed on from generation to generation, can be called the representative national culture. Especially in the rituals held in Spring Festival, in case of sacrifice activity, the "large waving song and dance" will be held and offer a sacrifice to the kings of 8 tribes. 
The song and dance is jointly held by performers from several villagers together in order to show their respect to the ancestors, and they will certainly dance Mao Gu Si dance.

Mao Gu Si is also called "hunting dance” in Chinese; in Tujia language, it is also called "Gu Si Bo Pu”; as a kind of ancient performing art of Tujia nationality, it has the performability of traditional Chinese opera, and it is called the "living fossil" of traditional Chinese opera by relevant Chinese experts. In terms of performance technique, Mao Gu Si is similar to virtual and comfortable property of opera, and the performance content is related to Tujia people's primitive life, such as fishing and hunting, and farming life; through expressing the daily living state by use of dance performance, the sacrifice dance in the grand sacrificial ceremony in the beginning of each year is formed.

Mao Gu Si is the most primitive way of dance performance of Tujia nationality, and it is created by Tujia ancestors in memory of remote ancestors' various kinds of living state in the time of eating the raw meat and drinking the blood, and it mainly simulates remote ancestors' labor state and living scene in ancient times. Although the content expressed via dance is shapeless artistic form, it is a display and supplement for the content to be expressed.

Except for Mao Gu Si song and dance, Tujia people divide "large waving” dance into 8 parts, that is, “start a journey”, “enter the hall”, “memorize the kings of 8 tribes”, "get married”, “migration and settlement”, “farming activity”, "generals and soldiers”, and "seeing-off”.

Corresponding to grand "large waving" dance, the "small waving” dance reflects Tujia people's daily life. In terms of sacrifice content, the content is mainly reflected via dance, and various kinds of waving actions are used to simulate agricultural work. In waving activities, Tujia people can sing folk songs in antiphonal singing or chorus. Although the "small waving” dance activity also belongs to sacrifice activity, it has an active atmosphere; it is mainly made in one clan beside Chieftain Temper.

As a cultural heritage of Tujia nationality, the waving dance is also a precious heritage of China's national culture. In 2008, in order to completely reserve Tujia waving dance as a kind of way of national cultural expression, this dance was listed into the list of China's intangible cultural heritage.

\section{Cultural characteristics of Tujia waving dance}

(I) Tujia people live in a form of ethnic group and form a folk-custom cultural circle. 
Although Tujia people have a habitation in southwest China, they are mainly distributed in Chongqing, Hubei, Hunan, Guizhou according to habitation situation; affected by geographical environment of Wuling Mountains, they live in a wide and scattered way. The blocked traffic creates relatively closed geographical environment and language environment. The different geographic climate makes Tujia people create their life by virtue of their hard work and braveness. This living environment also shapes Tujia people’s personality of active striving and struggling, and also forms a folk-custom cultural circle with Tujia style. The traditional folk dance waving dance has continued in Tujia people's life for thousands of years and has developed to now together with Chinese national culture, and it shows Tujia people's unique personality characteristics and ethnic character.

Since the ancient times, Tujia people always live in natural environment and natural villages; due to different administrative partition, the different dialect will form, which can reflect that different cultural contents are formed due to different living environment. Those cultural contents are limited to their nationality and internally inherited, which shows the survival state of Tujia people's unique folk-custom culture. In particular, Tujia culture can exist based on the family as a unit and it can be independently inherited, thus forming the self-individuality content and also forming the explanation system with different features.

Due to restraints of family system and influence of territory, natural villages, and language, Tujia people have a relatively closed cultural circle. The cultural clan inheritance makes Tujia language show cohesive force. Under such cultural environment, the continuous inheritance of waving dance is a result of collective participation. In the end or beginning of each year, Tujia people will organize a sacrifice which integrates various kinds of folk-custom culture of Tujia nationality and then becomes the reflection of Tujia people's cultural life.

Tujia waving dance has a long time, and it can be found from the characters recorded in Annals of Yongshun County Annals of Folk Custom: "with beautiful scenery beside Chieftain Temper, thousands of people gather together and have waving song and dance." This describes Tujia people's living state and shows distinct artistic features. The content of Tujia waving dance is also recorded in Annals of Yongshun in the period of Emperor Qianlong in Qing dynasty : "there is a waving hall in each village; from the $3^{\text {rd }}$ day to the $17^{\text {th }}$ day of lunar January, people gather together, beat gongs and drums, and dance and sing songs, and this 
custom still exist.” The expression form of waving dance is meticulously expressed, and the whole dance process condenses the unique Tujia folk-custom culture.

(II) Tujia waving dance has strong ornamental value.

From perspective of traditional folk custom, Tujia waving dance is mainly used for offering sacrifice to ancestors; with time development, while the sacrifice becomes a kind of cultural ritual, it gradually penetrates into civil activities and forms artistic culture. The waving dance has strong performance function and pays great attention to artistic expression, thus it has ornamental value. Tujia waving dance highlights "hand waving in same direction”, and it is mainly the waving of hands, and people wave their hands with rhythm. Upon hands waving, the waving shall be not beyond shoulder, but shall be beautiful and powerful. Certainly, there are some waving actions such as "beat the wave" and "comb the hairs". Although most of actions are imitated from Tong nationality, the action is smooth. As for the features of waving actions, there is difference in different regions and clans due to different way of production and labor. For example, in Mati Village of Longshan, people will wave their hands while walk transversely, and the two knees slightly vibrate with rhythm; as for Fengxi “waving dance”, people's hands wave at both sides of their body. The waving actions of Tujia waving dance all originate from daily life. The western Hunan Tujia people mainly live in hilly area, thus people shall walk in narrow meandering footpath everyday with heavy things on the body. Due to inconvenient action, they shall lean to one side upon walking. Those postures are also reflected in their waving dance.

Except for dance actions, the waving dance is integrated with song to form the artistic form of singing and dancing joyously. For example, at the beginning of waving dance, people will sing a shouting song; all lyrics are padding syllable and they are sung in a repeated way. While people listen to this song, they will consciously gather in waving hall to participate in waving activity. In case of "large waving”, the waving performance is led by Tima team (that is, Tujia wizards), with professional band and firecracker team, and those teams, including waving team and armored team, have accepted professional training.

\section{Fitness value of Tujia waving dance}

As a large-scale folk song and dance of Tujia nationality, it has collectivity and large scale. All people who participating in dance queue in a regular form and dance the dance according to 
musical rhythm or rhythm of song. The musical rhythm of waving dance is slow with large waving degree, which brings people a sense of elegance. According to the features of Tujia waving hands, the waving actions are diversified, and the waving postures include single waving, double waving, circle waving, and side waving, etc. While the body moves together with rhythm, the hands will wave rhythmically. The actions in whole process are natural, and each joint of whole body can be under coordinated movement, thus the ligament and muscle can be practiced well. It can be seen that the waving dance has fitness value.

Most of actions of Tujia waving dance originate from production and life. For example, among actions of waving dance, there are the action of "frog bathing under sunshine", the action of "chicken walking”, and the action of "seeding”, etc.; while large-scale waving action simulates those labor postures, it seems that the remote ancestors' scenes are showed. From sacrifice activity to entertainment, the functional value of waving dance steps from solemn ritual to people’s common life, and this dance becomes an entertainment activity. In waving dance performance, people can motivate their blood capillary, muscle fiber, and pulmonary alveolus through active participation. After there is sufficient oxygen in body, the body will obtain more nutrient substance and then people can improve their healthy condition. Furthermore, while people perform waving dance, they can be immersed into enjoyment of beauty and release their feelings, thus fully releasing people’s spiritual power.

\section{Conclusion}

In a word, among minorities in southwest China, Tujia nationality is one of the important minorities. Tujia waving dance with unique characteristics has been listed into the list of China's intangible cultural heritage. The waving dance has continued for thousands of years in Tujia culture; so far, it has become previous cultural heritage. Currently, Tujia waving dance not only exerts entertainment function, but also has the fitness value.

\section{Acknowledgments}

This paper is the research result of the project of Sichuan Provincial Department of Education: Research on Inheritance and Application of Dances in Qin-Ba Mountain Areas (Project No.: 13SB0021), and it is also the research result of 
China West Normal University "Research on Music and dance in Qin-Ba Mountain Areas” innovation team (Project No.: CXTD2012-12).

\section{References:}

[1] Liu Hui: Protection and Inheritance for Traditional Sports Culture of China's Intangible Cultural Heritage [J], Sports \& Science, 2007 (06).

[2] Yang Chuncai: Discussion on Positive Significance of Tujia Traditional Culture on Construction of Harmonious Society [J], Journal of Hubei University for Nationalities (Philosophy and Social Sciences), 2008 (01).

[3] Li Wei: Cultural Ecology and Cultural Inheritance of Tujia Waving Dance [J], Journal of South-Central University for Nationalities, 2007 (01).

[4] Yang Min, Chen Guangjiu: Research on Cultural Connotation and Cultural Value of Tujia Waving Dance [J], Journal of Wenzhou University (Natural Science), 2012. 33 (04).

[5] Liu Yan, Yuan Ge: Comprehensive Protection and Development on Tujia Waving Dance [J], Hunan Social Science, 2017 (06). 